\title{
Cavitation Bubble Collapse Monitoring by Acoustic Emission in Laboratory Testing
}

\author{
${ }^{1}$ Markku Ylönen*; ${ }^{1}$ Pentti Saarenrinne; ${ }^{1}$ Juha Miettinen; ${ }^{2}$ Jean-Pierre Franc; ${ }^{3}$ Marc Fivel \\ ${ }^{I}$ Tampere University of Technology, 33720 Tampere, Finland; ${ }^{2}$ Université Grenoble Alpes, CNRS, Grenoble INP, LEGI, 38000 \\ Grenoble, France; ${ }^{3}$ Université Grenoble Alpes, CNRS, Grenoble INP, SIMAP, 38000 Grenoble, France
}

\begin{abstract}
In order to investigate the potential of the acoustic emission technique in predicting cavitation erosion, laboratory tests were conducted in a high-speed cavitation tunnel. One face of a cylindrical stainless steel sample was subjected to an annular cavitation field created by the PREVERO cavitation tunnel [1]. Acoustic emission was measured from the back surface of the sample in order to detect impacts caused by cavitation bubble or cloud collapses. Cavitation aggressiveness was varied by changing the operating parameters of the cavitation tunnel. Two different operating points were compared. Collapsing cavitation bubbles lead to impacts towards the sample surface and they induce elastic waves in the material. A resonance type acoustic emission sensor with a resonance frequency of $160 \mathrm{kHz}$ captured these waves during the cavitation tests. The acoustic emission waveform was measured with a sampling frequency of $5 \mathrm{MHz}$. The sensor was mounted behind the sample using a wave-guide that maintained a transfer path for the elastic waves to travel from the impacted surface to the sensor. The elastic waves reaching the sensor were observed as distinguishable bursts in the acoustic emission waveform. Acoustic emission from cavitation impacts were estimated to be about 100 times stronger than acoustic emission from other sources, such as hydrodynamic events or machine vibration. This means that the signal was almost entirely induced by cavitation. The bursts contain multiple reflections that attenuate in time and that have a frequency content corresponding to the sensor frequency response. The bursts attenuate quickly enough not to overlap, as the cavitation events occur with a large enough temporal separation. The hypothesis in this study is that the maximum amplitude of the acoustic emission event voltage correlates with the strength of the cavitation bubble collapse impacting the surface. Voltage peak value counting was applied to the acoustic emission waveform data. As the bursts contain multiple amplitude peaks due to sensor resonance, an envelope function was fitted to the waveform for peak counting. Using this method, each counted voltage peak value is expected to correspond to a single cavitation impact event. The pulse distribution shows an exponential decrease with a decreasing voltage peak value rate as the peak voltage increases. This compares well with earlier studies, such as [2] and [3], where an exponential distribution of bubble collapse amplitudes was found. The results of this study prove acoustic emission as a direct and non-intrusive method that can be used to monitor cavitation impacts from outside of the cavitation field.
\end{abstract}

Keywords: cavitation impact detection; acoustic emission; cavitation intensity;

\section{Introduction}

The impact load induced by a cavitation bubble collapsing near a solid boundary has been studied by multiple methods, both experimental and computational. In a cavitating flow, the impact load determines material damage in a boundary caused by a single bubble or bubble cloud collapse. Another important factor in material damage is the impact frequency, as cavitation erosion tends to be a cumulative process [4-6]. The impact distribution that combines the impact frequency and amplitude of the cavitation impact loads is essential in determining the cavitation intensity of a flow.

Cavitation impact loads have been measured by various methods. Franc et al. [2] measured the impact loads in the PREVERO cavitation tunnel with conventional pressure sensors flush-mounted in the cavitation closure region. Hujer et al. [3] used PVDF pressure sensors, also flush-mounted in the same tunnel. Both of them observed exponential impact distributions. Hattori et al. [7] studied the impact pressures in an ASTM G-32 vibratory device and Okada et al. [8] used the same device to calibrate pressure sensors for a Venturi nozzle test. In the vibratory test, the impact distribution also follows an exponential law. Franc et al. [9] also observed the pits formed by cavitation impacts and they observed an exponential distribution in the pit size distribution. The pit shape factor or the ratio between pit depth and pit diameter increases with increasing cavitation intensity, meaning that larger impacts lead to deeper pits [10].

Several authors have studied acoustic emission (AE) as a method to detect and characterize cavitation and cavitation erosion. Boorsma and Fitzsimmons [11] created a cavitation monitoring method for ship rudders and propellers. Yongyong and Zaiyang [12] connected the AE-event energy to mass loss in an ASTM-G32 vibratory cavitation apparatus. Schmidt et al [13; 14] worked on a cavitation detection system based in AE on a prototype Kaplan turbine. They discovered that with properly placed sensors, cavitation leads to increase in AE root mean squared voltage value, event energy and fluctuation of both. Van Rijsbergen et al. [15] found that acoustic emission sensors in direct contact

*Corresponding Author, Markku Ylönen: markku.ylonen@tut.fi 
with a hydrofoil capture bubble collapses near the foil surface and with the cavitation impact towards the foil, but not those that occur in the flow far from the foil. These studies encourage further development of cavitation monitoring by AE.

In this study, the same cavitation tunnel as used by Franc et al. [2] and Hujer et al. [3] was fitted with acoustic emission sensors. The difference between previous studies with the tunnel in question is that the AE sensors are placed outside of the liquid flow by placing them in the backside of the sample. Voltage peak values corresponding to individual cavitation events were classified by their quantity and voltage. The goal was to produce similar distributions for two different operating conditions from the acoustic emission voltage peak values and to correlate these distributions with those of previous works.

\section{Experiments}

The experiments were carried out in the PREVERO high-speed cavitation tunnel in LEGI laboratory. The tunnel circulates water through a radially diverging test section. Water comes in to the test section through a $\emptyset 16 \mathrm{~mm}$ inlet nozzle and the flow stagnates in the middle of the test section where the sample is located and continues to diverge radially in a $2.5 \mathrm{~mm}$ thick channel. The samples are $20 \mathrm{~mm}$ thick cylindrical disks with a $100 \mathrm{~mm}$ diameter and placed so that the sample center is in the middle of the test section. Cavitation inception is located in the beginning of the radial section and cavitation closure is located between 21 and $26 \mathrm{~mm}$ radius of the disk when operating with the typical cavitation number $\sigma=0.9$. Cavitation number in PREVERO is defined in equation 1 :

$\sigma=\frac{P_{d}-P_{v}}{P_{u}-P_{d}}$

where $P_{d}$ is the pressure after the test section, $P_{u}$ is the pressure before the test section and $P_{v}$ is the liquid vapor pressure.

The acoustic emission sensors were fitted to the sample using a waveguide that is fixed with a screw thread to the sample. Figure 1(a) shows the sample disk flush mounted to the sample holder and the AE sensors fitted to the sample from behind. Figure 1(b) presents the test section without the sample and the sample holder. The sample and the holder were fastened to the test section so that from the inlet nozzle, the flow radially diverges to a $2.5 \mathrm{~mm}$ thick channel.
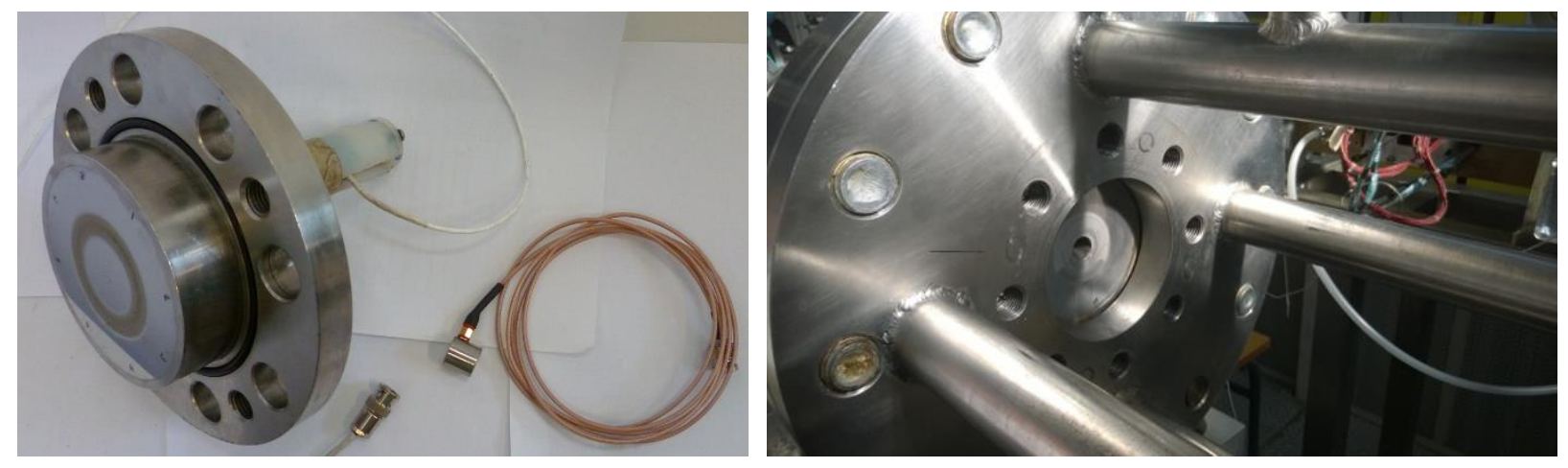

Figures 1(a) and 1(b). 1(a): Sample mounted to the sample holder and fitted with an AE sensor and a waveguide. 1(b): PREVERO test section opened and with the inlet nozzle in the middle. The sample is flush mounted so that it forms a part of the other wall in the test section.

Cavitation inception occurs at the outlet of the cylindrical nozzle, as the cross section area of the flow drops to 62.5 $\%$ of that of the inlet nozzle. Cavitation closure occurs further downstream as the cross section area and therefore the static pressure of the flow increase. Tunnel downstream tank is pressurized by nitrogen and upstream section by a pump linked to a frequency transformer. Downstream pressure varies from ambient pressure to around $3 \mathrm{MPa}$ and the maximum upstream pressure is $4 \mathrm{MPa}$. The cavitation tunnel is presented in more detail in $[1 ; 4]$.

The acoustic emission setup was a PAC PCI-2 two channel acquisition card fitted with PAC R15 and D9203b sensors and 20/40/60 preamplifiers. The R15 sensor is a resonance type sensor with a resonance frequency of $160 \mathrm{kHz}$. A 
band-pass filter from $100 \mathrm{kHz}$ to $400 \mathrm{kHz}$ was used. The D9203b sensor is a broadband sensor with a frequency range from $100 \mathrm{kHz}$ to $900 \mathrm{kHz}$. In this study, only the data acquired with the R15 sensor were analyzed. The samples were made of a stainless steel used in Francis turbines and the waveguides were made of steel.

In this study, two samples were mirror polished with successive diamond pastes and a colloidal silica suspension. One sample was subjected to cavitation erosion for 2 minutes with a $4 \mathrm{MPa}$ upstream pressure and the other for 6 minutes with a $2 \mathrm{MPa}$ upstream pressure. The $4 \mathrm{MPa}$ and the $2 \mathrm{MPa}$ upstream pressures correspond to $89.4 \mathrm{~m} / \mathrm{s}$ and $63.2 \mathrm{~m} / \mathrm{s}$ cavity reference velocities, respectively. Cavity reference velocity is defined as the velocity in the test section where pressure is assumed to be the vapor pressure. A more detailed explanation of cavity reference velocity is in [4]. Pits with this type of exposure covered roughly $10 \%$ of the surface of the cavitation closure area. The cavitation impacts were thus assumed to be hitting virgin material most of the time. Acoustic emission waveforms were acquired with a sampling frequency of $5 \mathrm{MHz}$ for the full duration of the tests.

\section{Peak value distribution by an envelope function}

Impacts in the cavitation sample surface were observed in the $\mathrm{AE}$ waveform as quickly rising voltage peaks that diminish exponentially. The AE waveforms were analyzed in time-voltage space. Wave propagation in the sample and the waveguide may affect the waveforms, but this effect is not considered in detail in this study. The frequency content of each cavitation burst or AE-event resembles that of the sensor frequency response, meaning that the impacts provoke sensor resonance. This means that the events are mostly short duration impacts compared to the sensor time scales. It is assumed that each voltage peak value in the waveform, with its resonance effects, corresponds to one cavitation impact towards the sample surface. The length of a cavitation bubble collapse is in the range of some microseconds up to some tens of microseconds $[2 ; 16]$. Through waveform observations, impact overlapping seems not to be significant.

With the assumption that each measured maximum in the voltage peak value corresponds to a single cavitation event, there is a need to filter out the sensor resonance effects when peak counting is applied. In most cases in this study, the acoustic emission waveform contains more or less isolated events with breaks between them. Each event has a distinct maximum or sometimes two or more maximums. To negate the resonance effects, an envelope function is fitted to the waveform. As the signal is approximately symmetric around zero volts, the absolute value of the signal is calculated. After this, the envelope function was fitted to the waveform. The envelope was calculated by spline interpolation over local maximums. The minimum distance between local peaks was set to $16 \mu \mathrm{s}$, which is about five times the distance between peaks resulting from sensor resonance. This value was found to be suitable through trial and error method. Figure 2 presents a typical $\mathrm{AE}$ waveform sample fitted with an envelope function.

*Corresponding Author, Markku Ylönen: markku.ylonen@tut.fi 


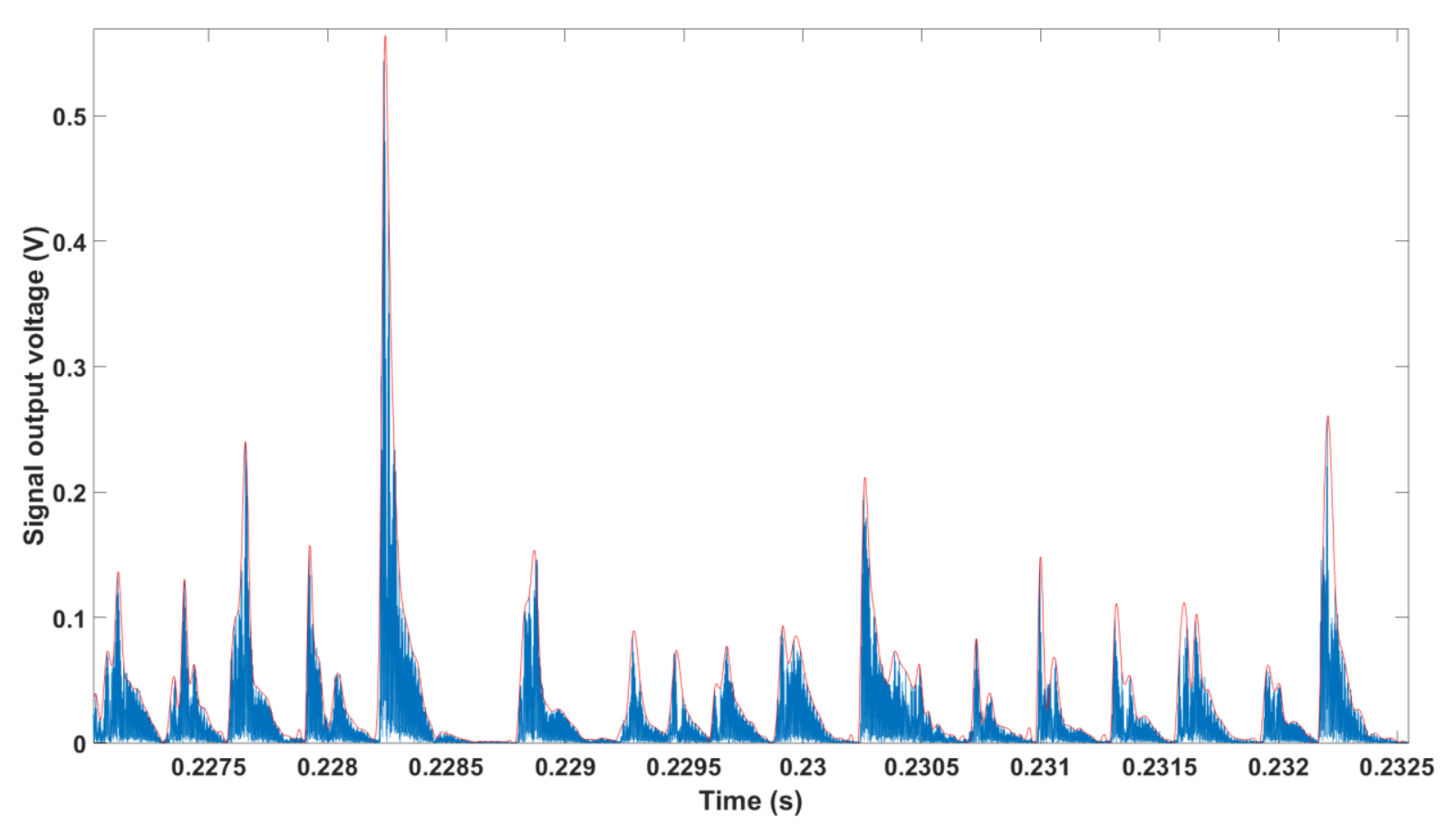

Figure 2. Typical AE waveform detail and the envelope function fitted to the waveform.

As observed in figure 2, the envelope follows the waveform, properly addressing the maximum values of each event. If the event contains two distinct peaks, it is assumed that two events are overlapping and both are taken into account. In the area outside the events, the envelope function may create false peaks due to noise and the formulation of the function. This effect is negated in the results, as events falling below a certain threshold are considered as either static noise or bubble collapses too weak to be erosive.

\section{Results}

The peak value distributions for both the $2 \mathrm{MPa}$ and the $4 \mathrm{MPa}$ upstream pressure tests are presented in figure 3 . The distributions are presented as cumulative so that each rate value represents the rate of voltage peak values larger than the corresponding voltage. The bin size for peak counting was $0.02 \mathrm{~V}$. The rate was expressed in [3] and [4] in counts per second per area, with the area being the sensor active area. In this study, the sensor captures all events occurring in the sample, so the active area cannot be properly defined. 


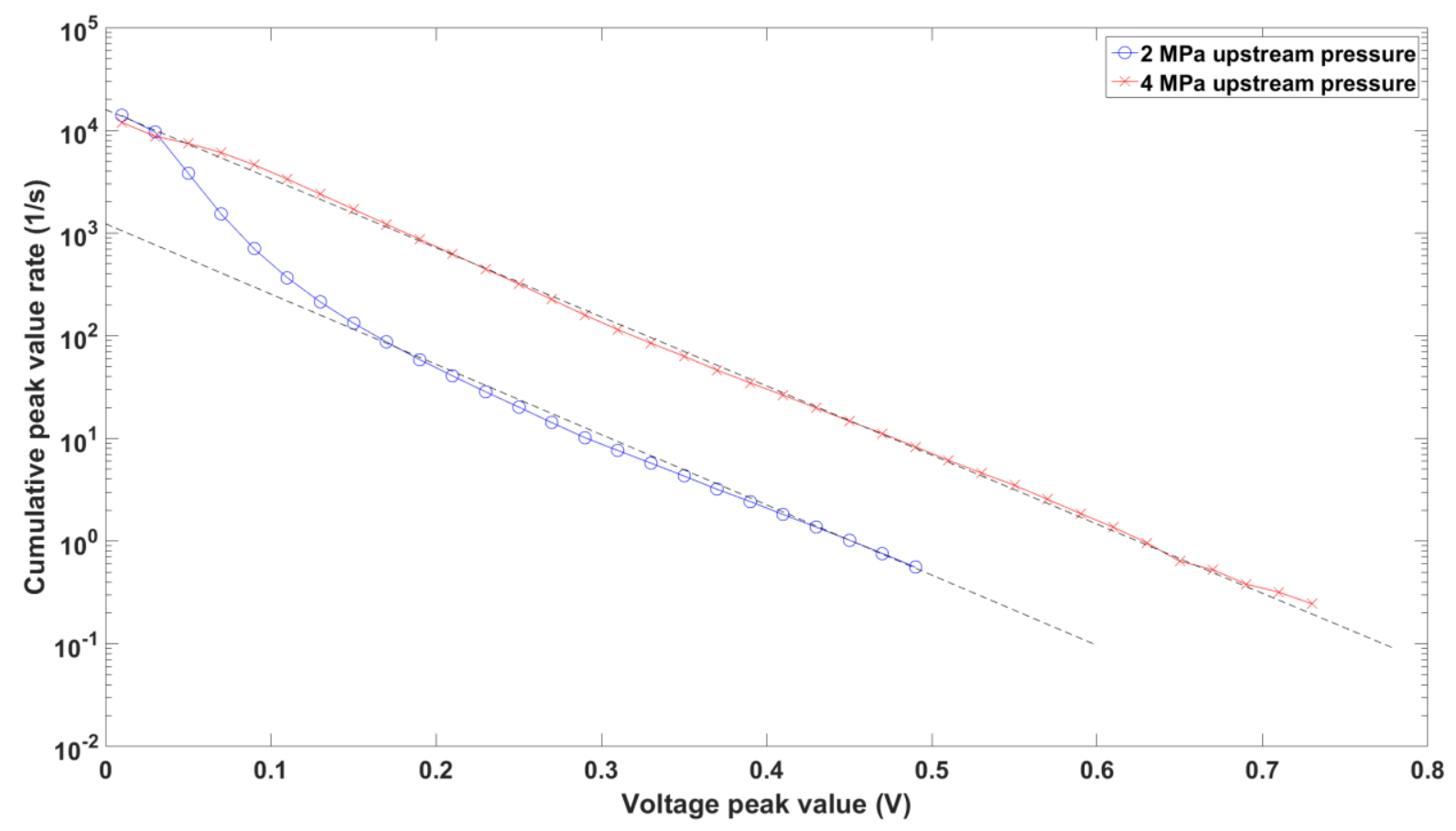

Figure 3. Cumulative peak rate vs. voltage peak value in linear-logarithmic scale. A linear fit was applied to the linear part of the curve.

The cumulative peak value distribution has a linear and a non-linear part in the linear-logarithmic scale. It was assumed that the non-linear part consists of static noise and insignificantly small bubble collapses. This study focuses only on the linear part of the distributions, corresponding to the expected exponential behavior of the cavitation impacts. The exponential law is presented in equation 2 :

$\dot{N}=\dot{N}_{0} e^{-\frac{U}{U_{0}}}$

where $\dot{N}$ is the peak rate, $\dot{N}_{0}$ is the reference peak rate, $U$ is the voltage peak value and $U_{0}$ is the reference voltage. The reference values $U_{0}$ and $\dot{N}_{0}$ are presented in table 1 .

\begin{tabular}{c|c|c}
$\begin{array}{c}\text { Upstream pressure/cavity } \\
\text { reference velocity }\end{array}$ & Reference peak rate $\dot{N}_{0}$ & Reference voltage $U_{0}$ \\
\hline $2 \mathrm{MPa} / 63.2 \mathrm{~m} / \mathrm{s}$ & $12321 / \mathrm{s}$ & $0.063 \mathrm{~V}$ \\
\hline $4 \mathrm{MPa} / 89.4 \mathrm{~m} / \mathrm{s}$ & $159581 / \mathrm{s}$ & $0.065 \mathrm{~V}$
\end{tabular}

Table 1. Cumulative distribution reference values

The linear parts of the cumulative distributions in figure 3 are essentially parallel. The reference voltages $U_{0}$ are calculated from the slope of the linear fits and they are found to be almost equal. The reference peak rate $\dot{N}_{0}$ follows the cavity reference velocity $V$ with a relation of $\dot{N}_{0} \sim V^{7.4}$. As the slope of the linear fit in linear-logarithmic scale does not change with the change of operating point, it means that the ratio between cumulative impact rates remains constant and independent of voltage peak value.

In this study, the connection between $\mathrm{AE}$ voltage peak values and the impact magnitude or impact damage is not addressed. Cavitation pitting in a virgin sample tends to produce pit sizes following a same type of exponential 
distribution as results in this and previous studies such as [9]. This fact is encouraging and shows that the results presented in this study somehow represent the physical phenomenon of cavitation pitting.

\section{Conclusion}

A method to monitor and characterize cavitation impacts by acoustic emission was presented. Acoustic emission was measured for two different operating conditions and envelope functions were fitted to the resulting waveforms. Voltage peak values were counted from the fitted envelope functions and cumulative distributions were calculated. Reference peak rates and reference voltages were calculated for both operating points. The reference voltage was about the same for both operating points and the reference peak rate had a relation of $\dot{N}_{0} \sim V^{7.4}$.

Using the presented method, it is concluded that the cumulative distributions for acoustic emission voltage peak rate value may be calculated for any operating condition by calculating the reference peak rate $\dot{N}_{0}$ and reference voltage $U_{0}$ and then applying the relation between reference peak rates, if the distribution for one point is known. To validate the results, multiple operating points should be tested. With the assumption that the relation holds as stated, the acoustic emission distributions can be used in estimating cavitation pitting in the cavitation tunnel. The advantage of the presented method is that it is non-intrusive, as it does not require direct access to the flow. Future studies will address these issues in more detail. These results show the potential of using acoustic emission in cavitation detection and characterization in laboratory testing and eventually in actual hydro machines.

\section{Acknowledgements}

The authors would like to thank Tekes (the Finnish Funding agency for Technology and Innovation), Fortum Power and Heat Oy, Sandvik Mining and Construction Oy, Valtra Oy and Teollisuuden Voima Oyj for funding the research and for providing technical support.

\section{References}

[1] The PREVERO cavitation erosion tunnel. Web page. Available (accessed 13/12/2017): http://www.legi.grenobleinp.fr/web/spip.php?article1265\&lang=fr.

[2] J. Franc, M. Riondet, A. Karimi, G.L. Chahine (2013). Impact Load Measurements in an Erosive Cavitating Flow. Journal of Fluids Engineering, 133(12).

[3] J. Hujer, J. Carrat, M. Müller, M. Riondet (2015). Impact load measurements with a PVDF pressure sensor in an erosive cavitating flow. Journal of Physics: Conference Series, 656(012051).

[4] J. Franc (2009), Incubation Time and Cavitation Erosion Rate of Work-Hardening Materials. Journal of Fluids Engineering, 131(2).

[5] J. Franc, G.L. Chahine, A. Karimi (2014). Pitting and Incubation period. in: K. Kim, G.L. Chahine, J. Franc, A. Karimi (ed.), 10.1007/978-94-017-8539-6. Springer Dordrecht Heidelberg, pp. 37-69.

[6] N. Berchiche, J. Franc, J. Michel (2002). A Cavitation Erosion Model for Ductile Materials. Journal of Fluids Engineering. 124(3).

[7] S. Hattori, T. Hirose, K. Sugiyama (2010). Prediction method for cavitation erosion based on measurement of bubble collapse impact loads. Wear. 269(7-8).

[8] T. Okada, Y. Iwai, S. Hattori, N. Tanimura (1995). Relation between impact load and the damage produced by cavitation bubble collapse. Wear. 184(2).

[9] J. Franc, M. Riondet, A. Karimi, G.L. Chahine (2012). Material and velocity effects on cavitation erosion pitting. Wear. $274-$ 275.

[10] S.C. Roy, J. Franc, M. Fivel (2015). Cavitation erosion: Using the material as a pressure sensor. Journal of Applied Physics. 118(16).

[11] A. Boorsma, P. Fitzsimmons (2009). Quantification of Cavitation Impacts with Acoustic Emissions Techniques. Proceedings of the 7th International Symposium on Cavitation (CAV).

[12] H. Yongyong, S. Zaiyang (2012). Experimental Research on Cavitation Erosion Detection Based on Acoustic Emission Technique. 30th European Conference on Acoustic Emission Testing \& 7th International Conference on Acoustic Emission. [13] H. Schmidt, O. Kirschner, S. Riedelbauch, J. Necker, E. Kopf, M. Rieg, G. Arantes, M. Wessiak, J. Mayrhuber (2014). Influence of the vibro-acoustic sensor position on cavitation detection in a Kaplan turbine. IOP Conference Series: Earth and Environmental Science. 22.

[14] H. Schmidt, O. Kirschner, S. Riedelbauch (2014). Cavitation measurements on a pump-turbine model. Journal of Physics: Conference Series. 656(1).

[15] M. van Rijsbergen, E. Foeth, P. Fitzsimmons, A. Boorsma (2012). High-Speed Video Observations and Acoustic-Impact Measurements on a NACA 0015 Foil. 8th International Symposium on Cavitation (CAV). 
[16] K. Kim, G. Chahine, J.-. Franc, A. Karimi (2014). Advanced Experimental and Numerical Techniques for Cavitation Erosion Prediction. 1st ed. Springer. 